$>$ La N-glycosylation est un mécanisme post-traductionnel ubiquitaire dans les cellules de mammifères dont les grandes étapes sont maintenant bien caractérisées. La découverte d'un groupe d'erreurs innées humaines affectant ce processus a fait évoluer les idées concernant la synthèse des $N$-glycosylprotéines $(N-G P)$ et a permis une meilleure compréhension de ce mécanisme complexe. Des mutations de plusieurs gènes impliqués dans la biosynthèse et la maturation des $N$ GP ont été caractérisées. En revanche, les liens entre les anomalies de glycosylation des N-GP et leurs répercussions cliniques n'ont pratiquement pas été explorés. Ce travail reste donc à accomplir et représente un défi qui pourrait, dans un certain nombre de cas, permettre d'envisager des voies de recherche thérapeutique. <

\section{Les anomalies congénitales de glycosylation des $\mathbf{N}$-glycosylprotéines}

Thierry Dupré, Grégory Lavieu, Stuart Moore, Nathalie Seta

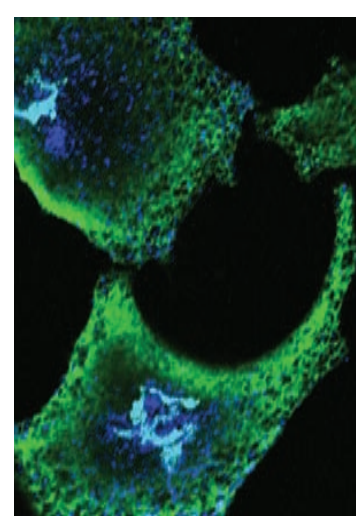

La N-glycosylation
Réseau de recherche sur les CDG I Inserm/AFM (4MR39F). T. Dupré, N. Seta : Service de Biochimie A, Hôpital Bichat, AP-HP, 16, rue Henri Huchard, 75877 Paris Cedex 18, France et Faculté de Pharmacie, Université Paris V, 75270 Paris Cedex 06, France. G. Lavieu, S. Moore : Inserm U.504, 16 , avenue Paul Vaillant Couturier, 94807 Villejuif Cedex, France.

Schématiquement, la synthèse des $N$-GP se nathalie.seta@ bch.ap-hop-paris.fr fait en deux temps. Tout d'abord, la chaîne peptidique d'une part, et l'oligosaccharide précurseur de la chaîne glycanique d'autre part, sont synthétisés de façon concomitante dans le réticumum endoplasmique (RE). L'oligosaccharide est transféré en bloc sur la chaîne peptidique. Dans un deuxième temps, l'oligosaccharide subit une maturation jusqu'à l'obtention de sa structure finale, caractéristique de la N-GP qui le porte.

$\mathrm{N}$-glycosylation et réticulum endoplasmique Comme le montre la Figure 1, les premières étapes impliquent la construction pas à pas d'un oligosaccharide lipide. Les monosaccharides (deux $\mathrm{N}$-acétylglucosamines [GlcNAc], neuf mannoses et trois glucoses) sont ajoutés séquentiellement, sur une ancre lipidique insérée dans la membrane du RE, le dolichyl-phosphate. Puis l'oligosaccharide est transféré en bloc, dans la lumière du RE, par l'oligosaccharyl transférase, sur l'asparagine d'un site consensus de $N$-glycosylation 
(-Asn-X-Ser/Thr-) d'une chaîne peptidique par une liaison $N$-glycosidique. Les $N$-glycanes «riches en mannose» (Figure 2A) jouent un rôle essentiel dans le repliement, le contrôle de qualité, la dégradation et l'adressage des glycosylprotéines [1, 2].

La conformation correcte des protéines néosynthétisées avant leur adressage vers leur site effecteur est en général acquise au niveau du RE. En effet, de cette conformation dépend en grande partie la fonctionnalité de la protéine. Un système de contrôle de qualité efficace garantit que seules les protéines correctement repliées poursuivent toutes les étapes de leur maturation, jusqu'à leur adressage, et ce par l'action des glucosidases I et II. La N-GP mal repliée est reglucosylée et interagit alors avec des lectines du RE, la calnexine et la calréticuline (Figure 2A), jusqu'à l'acquisition d'une conformation correcte.

\section{Transport des $\mathbf{N}-\mathrm{GP}$ et maturation dans l'appareil de Golgi}

La N-GP passe ensuite par transport vésiculaire, du RE vers l'appareil de Golgi par le biais de lectines, au moins pour certaines glycosylprotéines. Par exemple, la lectine ERGIC 53 [3] semble nécessaire pour un transport efficace des facteurs de coagulation V et VIII.

Dans les cellules de mammifères, la maturation golgienne amène les $\mathrm{N}$-glycanes à des structures dites «complexes», comprenant sur le pentasaccharide de base, des antennes formées de $N$-acétylglucosamine, de galactose, de fucose et d'acide sialique, mais

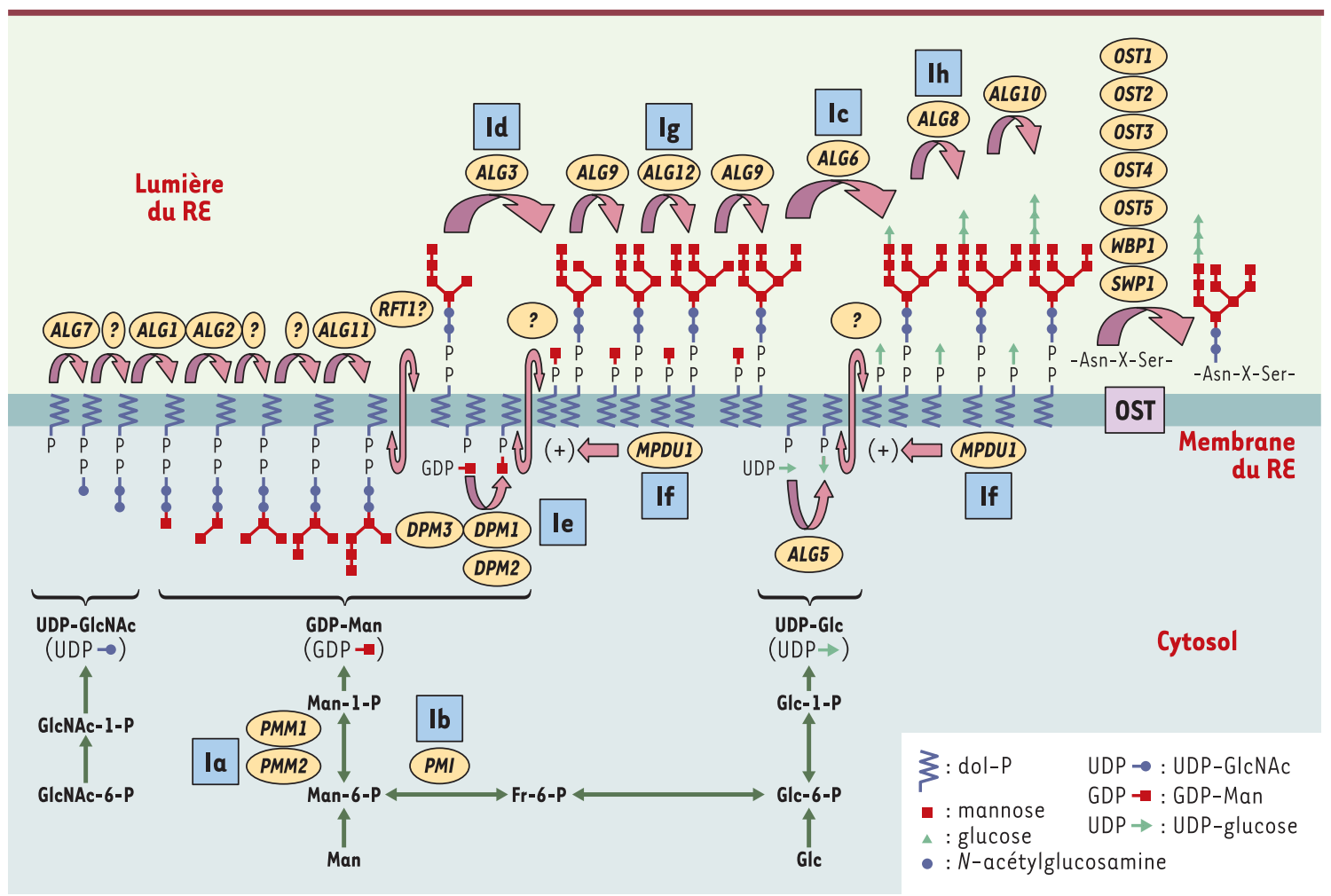

Figure 1. Synthèse de l'oligosaccharide lié au dolichol-pyrophosphate et CDG I (congenital disorder of glycosylation I). Les monosaccharides sont d'abord activés dans le cytosol avant d'être ajoutés (à gauche de la figure) au dolichol-phosphate (dol-P). Les sept premières réactions (qui utilisent comme donneurs I'UDP-N-acétylglucosamine [UDP-GIcNAc] et le GDP mannose [GDPMan]) se déroulent sur la face cytosolique de la membrane du réticulum endoplasmique (RE), alors que les ajouts ultérieurs de monosaccharides se situent dans la lumière du RE et utilisent le dolichol phospho-mannose (dol-P-Man) et le dolichol phosphoglucose (dol-P-Glc) comme donneurs. Le nom du gène codant pour l'enzyme responsable de chaque étape est indiqué dans un cercle jaune. L'oligosaccharyltransférase (OST), un complexe enzymatique comportant au moins sept protéines, transfère l'oligosaccharide $\mathrm{Glc}_{3} \mathrm{Man}_{9} \mathrm{GlcNAc}_{2}$ du dolichol pyrophosphate sur l'acide aminé asparagine d'une séquence consensus Asn-X-Ser/Thr d'un polypeptide en cours de biosynthèse dans la lumière du RE. Le déficit enzymatique de huit de ces étapes (carré bleu) est responsable des CDG (la à Ih). ALG : asparagine linked glycosylation; PMM : phosphomannomutase ; PMI : phosphomannose isomérase; DPM : dolichyl-phosphate mannosyl transférase 1 ; MPDU1 : mannose-phosphate dolichol utilization defect 1. 
dépourvues de glucose (Figures 2C, 2D, 3). Ces structures sont alors spécifiques d'une N-GP donnée, d'une part, et, d'autre part, de la localisation de sa synthèse en relation avec l'équipement enzymatique du tissu.

\section{Importance de la $\mathbf{N}$-glycosylation}

Les données concernant l'importance de la glycosylation proviennent essentiellement de trois sources. Tout d'abord, les études structurales, biochimiques et de biologie cellulaire ont permis de découvrir les rôles attribués à certains $N$-glycanes spécifiques dans divers «processus» cellulaires. Ainsi, l'étude de lignées mutantes de levures [4] et de cellules de mammifères [5], porteuses d'erreurs métaboliques de la $N$-glycosylation a fait largement progresser notre compréhension de la biosynthèse et des fonctions des $N$-glycanes. Puis,

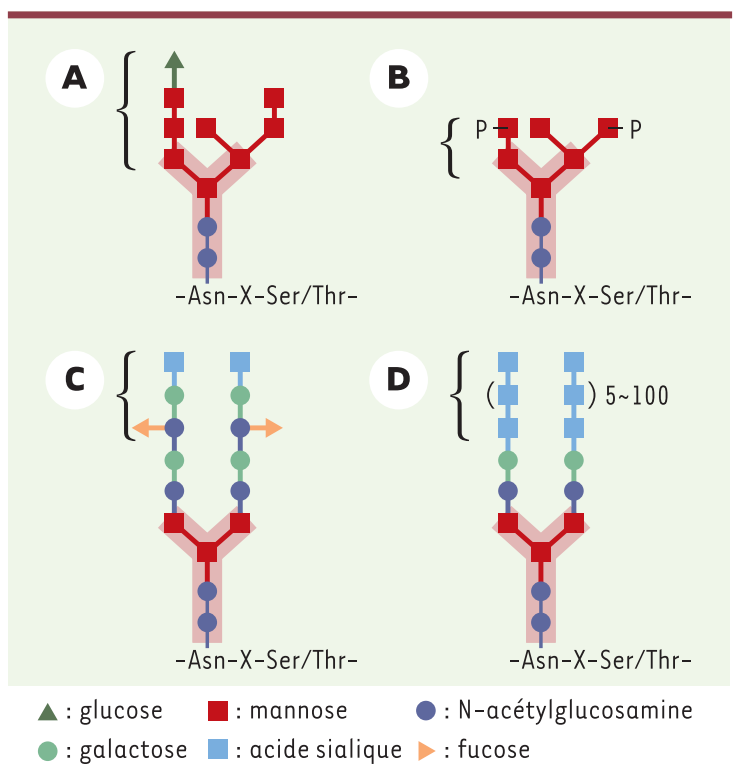

Figure 2. Exemples de structures $\mathbf{N}$-glycanes et leurs interactions avec des lectines. La structure pentasaccharidique de base (entourée d'une zone rose) est commune à tous les $N$ glycanes. A. oligosaccharide polymannosylé monoglucosylé (motif : glucosyl mannotriose ; lectine : calnexine, calréticuline) impliqué dans le repliement des glycosylprotéines dans la lumière du réticulum endoplasmique. $B$. oligosaccharide polymannosylé phosphorylé (motif : mannose 6-P (M6P) ; lectine : recepteurs $\mathrm{M} 6 \mathrm{P}$ ) responsable du ciblage et trafic des hydrolases lysosomales. $C$. oligosaccharide biantenné complexe (motif : sialyl Lewis $X$; lectine : P-sélectine) impliqué dans la diapédèse des leucocytes. $\boldsymbol{D}$. oligosaccharide biantenné porteur de motifs répétitifs d'acide sialique qui jouent un rôle dans la migration neuronale au cours du développement embryonnaire. à partir de modèles de souris transgéniques, il a été possible de déterminer l'importance de la présence, ou de l'absence, d'une structure glycanique particulière sur la physiologie de l'animal entier. L'ensemble de ces études indique que la biosynthèse des $N$-glycanes de type complexe est essentielle au développement embryonnaire. Enfin, la description d'un nombre croissant de maladies chez l'homme affectant la glycosylation, confirme les informations obtenues au niveau cellulaire, et apporte très souvent des éléments nouveaux aidant à comprendre les mécanismes complexes de la $N$-glycosylation.

\section{Anomalies de glycosylation des $\mathbf{N}$-GP}

Alors que les modifications acquises de structure de la glycosylation des $N$-GP sont peu nombreuses et portent essentiellement sur les sucres périphériques ou le nombre d'antennes, les erreurs innées de ce métabolisme, très variées, touchent l'ensemble de la glycosylation.

Parmi les premières maladies héréditaires décrites, la mucolipidose II (I-cell disease) a servi de «déclencheur» car elle associe un déficit enzymatique à une fonction essentielle de la glycosylation, l'adressage. Dans cette maladie, la majorité des hydrolases sont absentes des lysosomes mais sont retrouvées dans le milieu extracellulaire. On sait maintenant que cette affection est due à un déficit en GlcNAc-1-phosphotransférase du Golgi [6]. L'enzyme déclenche la synthèse du mannose 6-phosphate (Man-6-P) des chaînes $N$-glycaniques (Figure $2 B$ ), reconnu par son récepteur spécifique, le récepteur Man-6-P localisé dans l'appareil de Golgi. Cette lectine adresse la $N-G P$ portant le Man-6-P vers le lysosome. L'absence du Man-6-P ne permet pas le transport vers le lysosome, la N-GP restant alors dans le Golgi, puis étant excrétée dans le milieu extracellulaire.

Le LAD II (leukocyte adhesion deficiency) a permis d'illustrer l'importance des motifs saccharidiques dans l'interaction avec les sélectines. Les patients atteints de cette affection souffrent d'infections bactériennes récurrentes associées à une leucocytose périphérique, en relation avec une anomalie d'adhérence des leucocytes circulants [7]. Les cellules de ces patients produisent des N-GP à faible teneur en fucose, ce qui entraîne un déficit en antigènes de groupes sanguins $\mathrm{H} / \mathrm{ABO}$, sécrétoire et Lewis, ces derniers étant des ligands de sélectines requis dans la diapédèse des leucocytes (Figure 2C).

Un dépistage biochimique commun fondé sur l'anomalie de structure des $N$-GP sériques a permis la découverte récente de toute une série de nouvelles erreurs métabo- 
$(\rightarrow) \mathrm{m} / \mathrm{s}$ 1999, n 11 , p. 1202 liques [8] $(\rightarrow)$. Le groupe des CDG s'est ainsi constitué, comprenant les maladies associées à des erreurs innées métaboliques portant sur la $\mathrm{N}$-glycosylation (prévalence: $1 / 25000$ ). Bien que la présentation clinique varie d'un type de CDG à l'autre, une atteinte neurologique sévère associée à un retard mental et une hypotonie axiale est présente le plus souvent, à laquelle peuvent s'associer des atteintes multiviscérales graves [9].

Dès la découverte des premiers déficits enzymatiques, les glycobiologistes ont proposé une classification (Tableau I) tenant compte des grandes étapes de la synthèse des différents types de glycanes: synthèse de l'oligosaccharide lié au dolichol-phosphate jusqu'à son transfert sur la chaîne peptidique (CDG I) (Figure I) et maturation de la chaîne $N$-glycanique portée par la glycosylprotéine (CDG II) (Figure 3 ). En raison de la complexité de cette synthèse, on peut s'attendre à un nombre final important d'erreurs métaboliques différentes. Pour tous les CDG I actuellement connus, le déficit d'activité d'une protéine conduit à la synthèse d'une ou plusieurs espèces d'oligosaccharides - lipides tronqués, substrats d'affinité variable pour l'OST - qui s'accumulent dans le RE. Ce processus d'accumulation, délétère pour la cellule, permet d'identifier l'étape bloquante et de remonter à la protéine et au gène en cause. Les conséquences de ces accumulations sur l'homéostasie cellulaire et les dérèglements qui peuvent en résulter ont été encore très peu étudiées. Les répercussions sur d'autres voies métaboliques que celle de la synthèse des

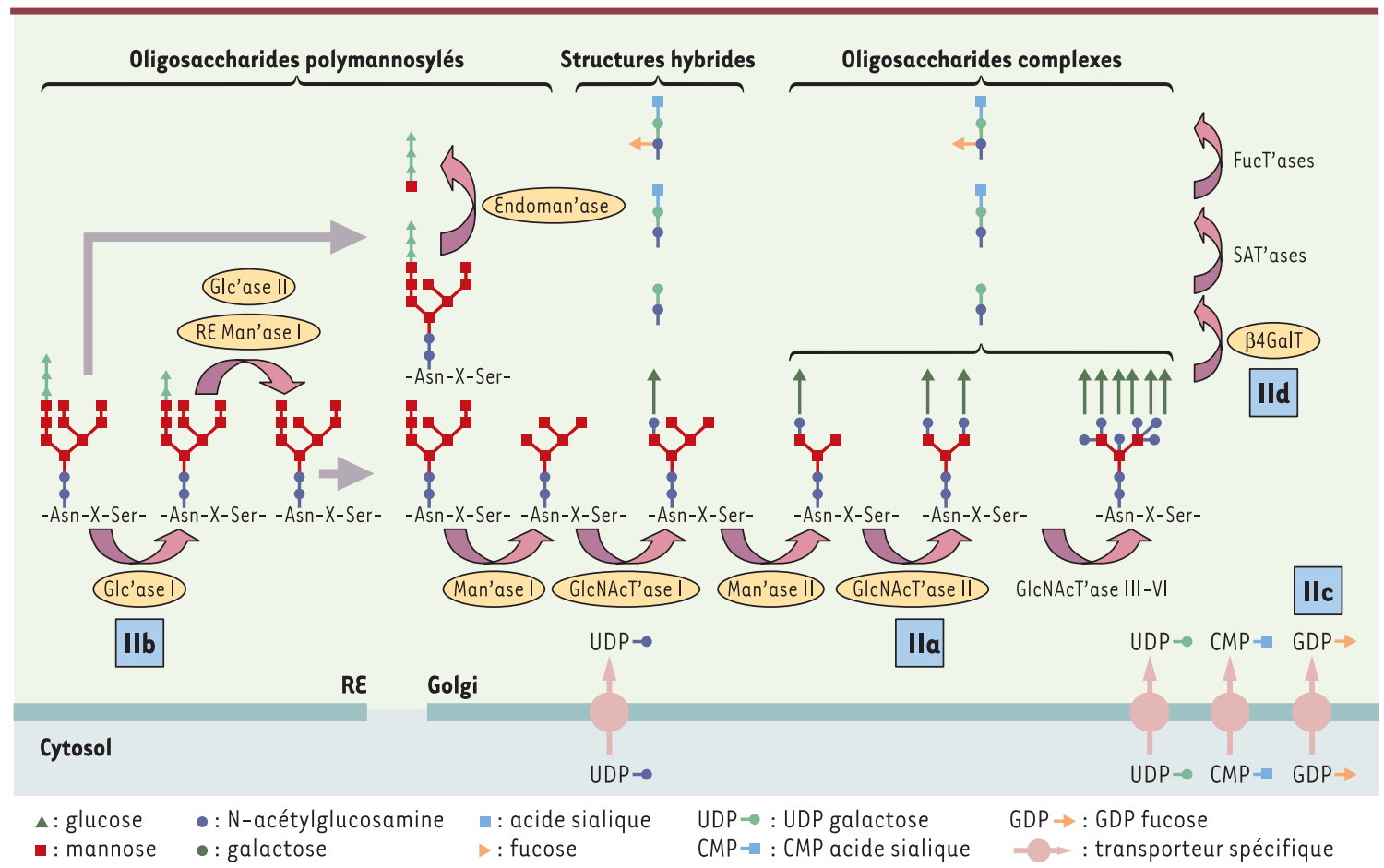

Figure 3. Maturation des N-glycanes et CDG II (congenital disorder of glycosylation II). La maturation des N-glycanes débute dans le RE par le clivage séquentiel de trois résidus de glucose et un résidu de mannose (glucodidase I, glucosidase II et mannosidase I du RE) et, après transport vésiculaire, se poursuit dans l'appareil de Golgi. Dans le cas de CDG IIb, qui correspond à un déficit en glucosidase I, les $\mathrm{N}$-glycanes triglucosylés apparaissent dans l'appareil de Golgi où ils subissent l'action de l'endomannosidase, qui libère le tétrasaccharide ( $\mathrm{Gl}_{3} \mathrm{Man}$ ) retrouvé dans les urines du patient. La maturation golgienne implique une démannosylation (mannosidase I) suivie par l'ajout de résidus $N$-acétylglucosamine avec le $N$-acétylglucosamine-UDP comme donneur (Glc$N A c$ transferases I, II, III), de résidus galactose avec I'UDP-galactose comme donneur ( $\beta$-1,4-galactosyltransferase), acide sialique avec l'acide sialique-CMP comme donneur (sialic acid transferase), fucose avec le GDP-fucose comme donneur (fucosyl transferase). Ces résidus entrent dans la lumière de l'appareil de Golgi grâce des transporteurs spécifiques. D'autres modifications, qui ne sont pas représentées dans cette figure, peuvent également avoir lieu (phosphorylation, sulfatation, polysialylation, etc.) et rendre les structures oligosaccharidiques golgiennes encore plus complexes. On aboutit ainsi à deux grandes classes d'oligosaccharides : les oligosaccharides polymannosylés et les olisaccharides complexes; des formes hybrides des deux classes sont également retrouvées. Quatre étapes de la maturation des $\mathrm{N}$-glycanes peuvent être concernées par un déficit enzymatique (indiqué par un carré bleu) responsable de CDG (Ila à IId). 


\begin{tabular}{|c|c|c|c|c|c|c|}
\hline $\begin{array}{l}\text { Type d'erreurs } \\
\text { métaboliques }\end{array}$ & CDG & $\begin{array}{l}\text { Anomalie } \\
\text { de glycosylation } \\
\text { des N-GP }\end{array}$ & $\begin{array}{l}\text { Métabolisme } \\
\text { déficitaire }\end{array}$ & Protéine & Gène & Réf. \\
\hline \multirow[t]{4}{*}{$\begin{array}{l}\text { 1. Production } \\
\text { et mobilisation } \\
\text { de sucre activé }\end{array}$} & CDG la & Abs $N$-GP & $\begin{array}{l}\text { Accumulation } \\
\text { inconstante de } \\
\mathrm{GICNAc}_{2} \mathrm{Man}_{5}\end{array}$ & PMM & PMM2 & [8] \\
\hline & CDG Ib & Abs N-GP & & PMI & MPI & [19] \\
\hline & CDG le & Abs N-GP & $\begin{array}{l}\text { Accumulation de } \\
\text { GlcNAc }_{2} \operatorname{Man}_{5}\end{array}$ & $\begin{array}{l}\text { Dol-P Man } \\
\text { synthase }\end{array}$ & $D P M I$ & [17] \\
\hline & CDG If & Abs N-GP & $\begin{array}{l}\text { Accumulation de } \\
\text { GlcNAc }_{2} \operatorname{Man}_{5}, \\
\text { GlcNAc }_{2} \operatorname{Man}_{9}, \\
\text { GlcNAc }_{2} \operatorname{Man}_{5} \mathrm{Glc}_{3} \\
\mathrm{GlcNAc}_{2} \operatorname{Man}_{9} \mathrm{Glc}_{3}\end{array}$ & Lec35 & MPDUI & [15] \\
\hline $\begin{array}{l}\text { 2. Transporteur } \\
\text { de sucre activé }\end{array}$ & $\begin{array}{l}\text { CDG \|c/ } \\
L A D \|\end{array}$ & $\begin{array}{l}\text { Défaut de fucosylation } \\
\text { des } N \text {-GP sériques }\end{array}$ & $\begin{array}{l}\text { Accumulation } \\
\text { de GDP-fucose }\end{array}$ & $\begin{array}{l}\text { Transporteur } \\
\text { du GDP-fucose }\end{array}$ & ORF-7 & [14] \\
\hline \multirow[t]{7}{*}{$\begin{array}{l}\text { 3. Glycosyl } \\
\text { transférase }\end{array}$} & CDG Ic & Abs N-GP & $\begin{array}{l}\text { Accumulation de } \\
\text { GlcNAc}_{2} \mathrm{Man}_{9}\end{array}$ & $\begin{array}{l}\text { Dol-P-Glc: } \\
\text { Dol-PP-GlcNAc } \\
\text { Man, }_{9} \alpha 1,3-G l c \\
\text { transférase }\end{array}$ & $h A L G 6$ & {$[26]$} \\
\hline & CDG Ig & Abs N-GP & $\begin{array}{l}\text { Accumulation de } \\
\text { GlcNAc }_{2} \operatorname{Man}_{7}\end{array}$ & $\begin{array}{l}\text { Dol-P-Man : } \\
\text { Dol-PP-GIcNAc } \\
\text { Man }_{7} \text {-Man- } \\
\text { transférase }\end{array}$ & $h A L G 12$ & [13] \\
\hline & CDG Ih & Abs N-GP & $\begin{array}{l}\text { Accumulation de } \\
\mathrm{GlcNAc}_{2} \mathrm{Man}_{9} \\
\mathrm{Glc}_{0-1}\end{array}$ & $\begin{array}{l}\text { Dol-P-Glc: } \\
\text { Dol-PP-GlcNAc } \\
\text { Man, Glc } \alpha 1,3-G l c \\
\text { transférase }\end{array}$ & $h A L G 8$ & [27] \\
\hline & CDG Id & Abs N-GP & $\begin{array}{l}\text { Accumulation de } \\
\text { GlcNAc }_{2} \operatorname{Man}_{5}\end{array}$ & $\begin{array}{l}\text { Dol-P-Man : } \\
\text { Man }_{5} \mathrm{Glc} \mathrm{NAc}_{2}- \\
\text { PP-Dol Man } \\
\text { transférase }\end{array}$ & $h A L G 3$ & [28] \\
\hline & $C D G \| a$ & $\begin{array}{l}\text { Chaîne monoantennée } \\
\text { des } N \text {-GP sériques }\end{array}$ & & $\begin{array}{l}\text { UDP-GIcNAc } \\
\alpha 6-D-M a n-\beta 1 \\
2-G I c N A c \\
\text { transférase II }\end{array}$ & MGAT2 & [12] \\
\hline & CDG $\| d$ & $\begin{array}{l}\text { Chaîne bi-antennée } \\
\text { monogalactosylée } \\
\text { et monosialylée } \\
\text { des } N \text {-GP sériques }\end{array}$ & & $\begin{array}{l}\text { UDP-Gal : } \\
\beta-G \mid c N A c_{2} \\
\beta-1,4- \\
\text { galactosyl } \\
\text { transférase I }\end{array}$ & B4GALTI & [29] \\
\hline & $\begin{array}{l}\text { l-cell } \\
\text { disease }\end{array}$ & $\begin{array}{l}\text { Hydrolases lysosomales } \\
\text { présentes dans le milieu } \\
\text { extracellulaire }\end{array}$ & & $\begin{array}{l}\text { GlcNAc-1- } \\
\text { phospho- } \\
\text { transférase }\end{array}$ & GNPT & [6] \\
\hline 4. Glycosidase & $C D G \| b$ & $\begin{array}{l}\text { Glc }(a l-2) G I c(a l-3) \\
\text { Glc }(a l-3) \text { Man présent } \\
\text { dans les urines }\end{array}$ & & Glucosidase I & GLSI & {$[30]$} \\
\hline
\end{tabular}

Tableau I. Les anomalies congénitales de glycosylation (congenital disorders of glycosylation ou CDG). N-GP: N-glycosylprotéines ; Abs N-GP : absence des chaînes glycaniques des N-GP sériques; PMM : phosphomannomutase ; PMI : phosphomannose isomérase ; GlcNac : N-acétylglucosamine ; Man : mannose ; Glc : glucose ; MPDUl : mannose-P-dolichol utilization defect 1. 
N-GP sont donc à explorer. Néanmoins, les travaux menés à ce jour contribuent à une meilleure connaissance de la $N$-glycosylation.

\section{Nouveaux métabolismes, nouveaux gènes}

Le CDG la, historiquement le premier décrit, est dû à un déficit en phosphomannomutase (PMM), enzyme au cœur du métabolisme du mannose, tout comme la phosphomannose isomérase (PMI), déficitaire dans le CDG Ib. De nombreux indices ont laissé supposer que le métabolisme du mannose, alors inconnu chez l'homme, était en cause dans ces CDG [8]. Ainsi, les gènes de la PMM, $P M M 1$ et $P M M 2$, ainsi qu'un pseudo-gène, ont été identifiés [10]. Un transport membranaire facilité spécifique du mannose a été mis en évidence à la surface de tous les types cellulaires étudiés. Enfin, il a été démontré que la source prépondérante de mannose pour la synthèse des N-GP n'était pas le glucose mais le mannose [11]. L'identification de nouvelles formes de CDG a conduit à la mise en cause de nouveaux gènes humains, connus ou non chez la levure, comme hALG5, hALG6, hALG12, ou encore le gène du transporteur du GDP-fucose [12-14]. Cependant, un grand nombre d'aspects de la glycosylation ne sont pas encore compris. Ainsi, les flippases responsables de la translocation des structures synthétisées sur la face cytosolique du RE restent à préciser [15], tout comme le rôle des protéines associées, telles que la protéine RFTl [16]. Il est également évident que les glycosyltransférases reçoivent l'assistance de protéines dont certaines sont identifiées comme MPDUl (mannose-Pdolichol utilization defect 1) ou encore DPM2 (dolichylphosphate mannosyl synthase 2) et DPM3 (Figure 1) mais dont les fonctions ne sont pas toutes clairement établies.

\section{Conséquences des anomalies de synthèse ou d'utilisation des monosaccharides activés}

Les anomalies de synthèse et mobilisation des monosaccharides activés (CDG la, Ib, le, If, IIc) sont lourdes de conséquences. Un exemple est celui du GDP-fucose dans le CDG IIc/LAD II et de ses effets sur la diapédèse des leucocytes [14]. Les troubles métaboliques peuvent affecter la glycosylation dans son ensemble et ne pas se limiter seulement à la $\mathrm{N}$-glycosylation. Certains des monosaccharides activés utilisés dans la synthèse des $N$-glycanes servent également de substrats pour la synthèse d'autres types moléculaires comme les ancres glycosyl-phosphatidyl-inositol (GPI) ou les glycolipides. Ainsi, le défaut de synthèse de dol-P-Man (CDG le) conduit à un défaut de production de protéines fixées à la membrane par une ancre GPI, avec une diminution franche de l'antigène $C D$ 59 à la surface des fibroblastes de patient CDG le [17].
Par ailleurs, la synthèse des glycosphingolipides dans des fibroblastes de patients CDG la est accrue, comparée à celle des témoins et, de plus, leur dégradation est réduite [18]. Cette anomalie est liée à un détournement de l'utilisation de ces monosaccharides vers les glycosphingolipides du fait de la synthèse déficiente des $N$-glycanes. Ces résultats mettent en évidence la complexité et les intrications des voies de biosynthèse au sein du processus de glycosylation au sens large.

Dans certains cas, ces passerelles métaboliques peuvent constituer un recours et permettre la mise en place d'un traitement par supplémentation orale en mannose pour le CDG Ib [19].

\section{Adaptation des cellules aux troubles de glycosylation}

Connaissant l'importance des $\mathrm{N}$-glycanes dans la fonctionnalité des $N$-GP, les troubles constatés dans les CDG, au niveau cellulaire ou de l'organisme entier, ne semblent pas aussi sévères que l'on pourrait s'y attendre. Ainsi, les images de fibroblastes de patients CDG I obtenues par microscopie électronique montrent une organisation cellulaire conservée à l'exception d'un RE dilaté [20]. Par ailleurs, les défauts majeurs constatés lors de la synthèse précoce des N-GP n’ont de répercussions structurales que sur un nombre limité de protéines, d'origine hépatique [21]. Enfin, le degré d'hypoglycosylation des $N$-GP sériques peut varier notablement d'un patient à l'autre [22].

Cette correction des défauts de glycosylation pourrait faire appel à deux processus complémentaires : d'une part, la persistance d'une activité résiduelle de la protéine mutée, couplée à la très forte affinité de l'oligosaccharyl transférase pour son substrat normal et, d'autre part, un mécanisme d'adaptation via la stimulation de I'UPR (unfolded protein response). Dans le cas des CDG I, le transfert d'oligosaccharides tronqués ou l'absence de transfert sur des sites normalement glycosylés entraînent le mauvais repliement d'un certain nombre de protéines et donc un stress constitutif au niveau cellulaire. En réponse, les cellules eucaryotes déclenchent I'UPR associée à I'ERAD (endoplasmic reticulum associated degradation) [23]. Ces mécanismes permettent de maintenir l'équilibre cellulaire en limitant l'accumulation de protéines dans la lumière $d u R E$. La $N$-glycosylation qui participe au repliement des $N$-GP et le stress du RE semblent liés: une activation constitutive de I'UPR a été décrite dans une lignée cellulaire CHO déficitaire en DPM (analogue au CDG le) [24] et la tunicamycine, un inhibiteur de la $N$-glycosylation, induit I'UPR. De plus, un RE dilaté a été décrit dans des fibroblastes issus de patients CDG-la [20]. Ces différentes observations suggèrent un lien entre la maladie 
et le stress du RE, qui peut déclencher une signalisation conduisant à la mort cellulaire.

Finalement, connaissant l'importance des N-GP dans le développement cérébral (Figure 2D), l'observation d'une hypoplasie cérébelleuse parmi les atteintes neurologiques des CDG, suggère un lien entre la perturbation de la $\mathrm{N}$-glycosylation et le comportement neuronal, soit au travers d'une mort cellulaire précoce, soit par un défaut de migration des neurones. En effet, un lien a été établi entre plusieurs maladies neurodégénératives et la mort apoptitique des cellules [25].

\section{Conclusions}

Malgré le retard pris dans l'exploration de la synthèse la partie glycanique des $N$-GP par rapport à d'autres métabolismes, les morceaux du puzzle s'assemblent progressivement. Cependant, l'intégration des données acquises en recherche fondamentale ne permet pas encore de comprendre les mécanismes physiopathogéniques des atteintes cliniques chez les patients souffrant d'anomalies congénitales de glycosylation. $\diamond$

\section{SUMMARY}

\section{Inherited disorders of protein glycosylation}

Protein $\mathrm{N}$-glycosylation is a widely occurring and vital posttranslational modification in mammalian cells. Although the molecular machinery that is involved in the biosynthesis of these glycoconjugates has been largely identified, the recent discovery of a family of rare inborn diseases in which glycoproteins are abnormally glycosylated has both changed some of our ideas concerning glycoprotein biosynthesis, and given us new insights into this complex process. Advances in the diagnosis of the congenital disorders of glycosylation are well under way and mutations in several of the genes involved in the biosynthesis and maturation of $\mathrm{N}$-linked glycans have been shown to underlie these diseases. By contrast, the chain of events that lead from faulty protein glycosylation to the often severe clinical presentation is an as yet unexplored aspect of these metabolic disorders, and represents a challenge for the future. $\diamond$

\section{RÉFÉRENCES}

1. Moore SE. Oligosaccharide transport : pumping waste from the $\varepsilon R$ into lysosomes. Trends Cell Biol 1999; 9 : 441-6.

2. Helenius $A$, Aebi M. Intracellular functions of $N$-linked glycans. Science $2001 ; 291: 2364-9$.
3. Nichols WC, Seligsohn U, Zivelin A, et al. Mutations in the ER-Golgi intermediate compartment protein ERGIC- 53 cause combined deficiency of coagulation factors V and VIII. Cell $1998 ; 93: 61-70$.

4. Burda P, Aebi M. The dolichol pathway of $N$-linked glycosylation. Biochim Biophys Acta 1999; 1426 : 239-57.

5. Stanley P, Sallustio S, Krag SS, Dunn B. Lectin-resistant CHO cells : selection of seven new mutants resistant to ricin. Somat Cell Mol Genet 1990; 16: 211-23.

6. Hickman S, Neufeld EF. A hypothesis for I-cell disease : defective hydrolases that do not enter lysosomes. Biochem Biophys Res Commun $1972 ; 49: 992-9$.

7. Anderson DC, Springer TA. Leukocyte adhesion deficiency : an inherited defect in the Mac-1, LFA-1, and p150,95 glycoproteins. Annu Rev Med 1987 ; $38: 175-94$.

8. Seta N, Dupre T, Cormier-Daire V, et al. Le carbohydrate-deficient glycoprotein syndrome de type I: un nouvel éclairage sur le métabolisme du mannose. Med Sci (Paris) 1999; 15 : 1202-10.

9. Jaeken J, Matthijs G. Congenital disorders of glycosylation. Annu Rev Genomics Hum Genet $2001 ; 2$ : 129-51.

10. Schollen $\varepsilon$, Pardon $\varepsilon$, Heykants $L$, et al. Comparative analysis of the phosphomannomutase genes PMM1, PMM2 and PMM2psi : the sequence variation in the processed pseudogene is a reflection of the mutations found in the functional gene. Hum Mol Genet 1998 ; 7 : 157-64.

11. Panneerselvam K, Freeze HH. Mannose corrects altered $\mathrm{N}$-glycosylation in carbohydrate-deficient glycoprotein syndrome fibroblasts. J Clin Invest 1996; 97 : 1478-87.

12. Schachter $\mathrm{H}$. Congenital disorders involving defective $\mathrm{N}$-glycosylation of proteins. Cell Mol Life Sci 2001 ; 58 : 1085-104.

13. Chantret I, Dupre T, Delenda C, et al. Congenital disorders of glycosylation (CDG) type Ig is defined by a deficiency in dolichol-Pmannose : Man7GIcNAc2-PP-dolichol mannosyltransferase. J Biol Chem $2002 ; 277: 25815-22$.

14. Lubke T, Marquardt T, Etzioni A, et al. Complementation cloning identifies CDG-IIc, a new type of congenital disorders of glycosylation, as a GDP-fucose transporter deficiency. Nat Genet $2001 ; 28: 73-6$.

15. Schenk B, Fernandez F, Waechter CJ. The ins(ide) and out(side) of dolichyl phosphate biosynthesis and recycling in the endoplasmic reticulum. Glycobiology $2001 ; 11: 61 \mathrm{R}-70 \mathrm{R}$.

16. Helenius J, Ng DT, Marolda CL, et al. Translocation of lipid-linked oligosaccharides across the $\varepsilon R$ membrane requires Rftl protein. Nature $2002 ; 415: 447-50$.

17. Imbach T, Schenk B, Schollen $\varepsilon$, et al. Deficiency of dolichol-phosphatemannose synthase- 1 causes congenital disorder of glycosylation type le. J Clin Invest $2000 ; 105: 233-9$.

18. Sala G, Dupre T, Seta N, et al. Increased biosynthesis of glycosphingolipids in congenital disorder of glycosylation la (CDG-la) fibroblasts. Pediatr Res $2002 ; 52: 645-51$.

19. de Lonlay P, Cuer M, Vuillaumier-Barrot $S$, et al. Hyperinsulinemic hypoglycemia as a presenting sign in phosphomannose isomerase deficiency : a new manifestation of carbohydrate-deficient glycoprotein syndrome treatable with mannose. J Pediatr 1999 ; 135 : 379-83.

20. Marquardt T, Ullrich K, Zimmer P, et al. Carbohydrate-deficient glycoprotein syndrome (CDGS)-glycosylation, folding and intracellular transport of newly synthesized glycoproteins. Eur J Cell Biol 1995 ; $66: 268-73$.

21. Dupre T, Barnier A, de Lonlay P, et al. Defect in $N$-glycosylation of proteins is tissue-dependent in congenital disorders of glycosylation la. Glycobiology $2000 ; 10$ : 1277-81.

22. Dupre $T$, Cuer $M$, Barrot $S$, et al. Congenital disorder of glycosylation la with deficient phosphomannomutase activity but normal plasma glycoprotein pattern. Clin Chem 2001 ; 47 : 132-4.

23. Chevet $\varepsilon$, Cameron PH, Pelletier MF, et al. The endoplasmic reticulum: integration of protein folding, quality control, signaling and degradation. Curr Opin Struct Biol $2001 ; 11$ : 120-4.

24. Foulquier $F$, Harduin-Lepers $A$, Duvet $S$, et al. The unfolded protein response in a dolichyl phosphate mannose-deficient Chinese hamster ovary cell line points out the key role of a demannosylation step in the quality-control mechanism of $N$-glycoproteins. Biochem J 2002 ; $362: 491-8$.

25. Nakagawa T, Zhu H, Morishima N, et al. Caspase-12 mediates endoplasmic-reticulum-specific apoptosis and cytotoxicity by amyloidbeta. Nature $2000 ; 403: 98-103$. 
26. Korner C, Knauer R, Holzbach U, et al. Carbohydrate-deficient glycoprotein syndrome type $V$ : Deficiency of dolichyl-P-

Glc:Man9GIcNAc2-PP-dolichyl glucosyltransferase. Proc Natl Acad Sci USA $1998 ; 95: 13200-5$

27. Chantret I, Dancourt J, Dupre T, et al. A deficiency in dolichyl-Pglucose: Glc1Man9GIcNAc2-PP-dolichyl alpha3-glucosyltransferase defines a new subtype of congenital disorders of glycosylation. J Biol Chem 2003: $278:$ 9962-71.

28. Korner C, Knauer R, Stephani U, et al. Carbohydrate deficient glycoprotein syndrome type IV : deficiency of dolichyl-P-

Man:Man(5)GIcNAc(2)-PP-dolichyl mannosyltransferase. Embo J 1999; 18: 6816-22.
29. Hansske B, Thiel C, Lubke T, et al. Deficiency of UDP-galactose : $N$ acetylglucosamine beta-1,4-galactosyltransferase I causes the congenital disorder of glycosylation type Ild. J Clin Invest 2002 ; 109 : 725-33.

30. De Praeter CM, Gerwig GJ, Bause $\varepsilon$, et al. A novel disorder caused by defective biosynthesis of $\mathrm{N}$-linked oligosaccharides due to glucosidase I deficiency. Am J Hum Genet 2000 ; 66 : 1744-56.

\section{TIRÉS À PART}

T. Dupré 